\title{
COMMON FIXED POINT THEOREMS IN COMPLETE METRIC AND PROBABILISTIC METRIC SPACES
}

\author{
Mila Stojakovic
}

In this paper several common fixed point theorems for four continuous mappings in Menger and metric spaces are proved. These mappings are assumed to satisfy some generalizations of the contraction condition.

\section{Introduction.}

A Menger space is a space in which the concept of distance is considered to be probabilistic, rather then deterministic. For a detailed discussion of Menger spaces and their applications we refer to Schweizer and Sklar [9]. The theory of Menger spaces is of fundamental importance in probabilistic functional analysis. Recently, some fixed point theorems for mappings in Menger spaces have been proved by several authors: G. Bocsan, A.F. Bharucha-Raid, S.S. Chang, Gh. Constantin, O. Hadzić and others (see [6]).

Since every metric space is a Menger space, all results in Menger space, with some modifications, can be used in metric spaces.

3. Preliminaries.

Let $R$ denote the reals and $\boldsymbol{R}^{+}=\{x \in \mathbb{R}: x \geq 0\}$. A mapping

Received Il July 1986.

Copyright Clearance Centre, Inc. Serial-fee code: 0004-9729/87 $\$ \mathrm{~A} 2.00+0.00$. 
$F: R \rightarrow \mathbb{R}^{+}$is called a distribution function if it is non-decreasing, left continuous with inf $F=0$ and $\sup F=1$. We will denote by $\Delta$ the set of all distribution functions. A commutative, associative and nondecreasing mapping $t:[0,1] \times[0,1]+[0,1]$ is a $T$-norm if and only if $t(a, 1)=a$ for all $a \in[0,1]$ and $t(0,0)=0$.

DEFINITION 1. A Menger space is a triple $(X, F, t)$ where $X$ is a set, $F$ is a mopping from $X \times X$ into $\Delta$ and $t$ is a T-norm. We shall denote the distribution function $F(x, y)$ by $F_{x, y}$ and $F_{x, y}(\varepsilon)$ will represent the value of $F_{x, y}$ at $\varepsilon \in \mathbb{R}$. The function $F_{x, y}$, $x, y \in X$, are assumed to satisfy the following conditions:

1. $F_{x, y}(\varepsilon)=1$ for $\varepsilon>0$ if and only if $x=y$.

2. $F_{x, y}(0)=0$, for all $x, y \in X$.

3. $F_{x, y}=F_{y, x}$, for all $x, y \in X$.

4. $F_{x, y}(\varepsilon+\delta) \geq t\left(F_{x, z}(\varepsilon), F_{z, y}(\delta)\right)$, for alz $x, y, z \in X$.

Throughout this paper $H$ will denote the specific distribution function defined by

$$
H(\varepsilon)= \begin{cases}0 & \varepsilon \leq 0 \\ 1 & \varepsilon>0 .\end{cases}
$$

The concept of neighbourhoods in Menger space was introduced by Schweizer and sklar [9]. If $x \in X, \varepsilon>0$ and $\lambda \in(0,1)$, then an $(\varepsilon, \lambda)-$ neighbourhood of $x$, called $U_{x}(\varepsilon, \lambda)$, is defined by

$$
U_{x}(\varepsilon, \lambda)=\left\{y \in X: F_{x, y}(\varepsilon)>1-\lambda\right\} .
$$

If $t$ is continuous, then $(X, F, t)$ is a Hausdorff space in the topology induced by the family $\left\{U_{x}(\varepsilon, \lambda): x \in X, \varepsilon>0, \lambda \in(0,1)\right\}$ of neighbourhoods.

DEFINITION 2. A set $M \subseteq X$ is called probabilistically bounded if and on zy if

$$
\sup _{\varepsilon>0} \inf _{x, y \in M} F_{x, y}(\varepsilon)=1
$$




\section{Fixed point Theorem in Menger space.}

THEOREM 1. Let $(X, F, t)$ be a complete Menger space with a continuous T-norm $t$ and let $h: X \rightarrow X, k: X \rightarrow X, f: X \rightarrow h(X)$ and $g: X+k(X)$ be continuous moppings such that $f$ conmutes with $k$ and $g$ commutes with $h$. Further, suppose that for all $x, y \in X$ and for all $\varepsilon>0$ the following inequality holds

$$
F_{f x, g y}(\varepsilon) \geq F_{k x, h y}(\phi(\varepsilon)),
$$

where $\phi: \mathbb{R}^{+} \rightarrow R^{+}$is an increasing function such that $\lim _{n \rightarrow \infty} \phi^{n}(t)=\infty$ for all $t>0$. If there exists $x_{1} \in X$ such that the sequence $\left\{y_{n}\right\}_{n \in N}$ formed by

$$
\begin{aligned}
& y_{2 n-1}=g x_{2 n-1}=k x_{2 n}, \\
& y_{2 n}=f x_{2 n}=h x_{2 n+1}, n \in N
\end{aligned}
$$

is probabilistically bounded, then there exists a unique conmon fixed point for the mappings $f, g, h$ and $k$.

Proof. Since $f: X \rightarrow h(X)$ and $g: X \rightarrow k(X)$ we can form the sequence $\left\{y_{n}\right\}_{n \in N}$ as was noted above. First, we shall show that $\left\{y_{n}\right\}_{n \in N}$ is a Cauchy sequence.

In order to prove that, we shall show that

$$
\lim _{\substack{m \rightarrow \infty \\ p \rightarrow \infty}} F_{y_{m}, y_{p}}(\varepsilon)=H(\varepsilon) \text {, for every } \varepsilon \in \mathbb{R} \text {. }
$$

If $m=2 i$ and $p=2 j-1$ (let $j>i$ ) then we have

$$
\begin{aligned}
& F_{y_{2 i}, y_{2 j-1}}(\varepsilon)=F_{f x_{2 i}, g x_{2 j-1}}(\varepsilon) \geq F_{k x_{2 i}, h x_{2 j-1}}(\phi(\varepsilon)) \\
& =F_{f x_{2 j-2}, g x_{2 i-1}}(\phi(\varepsilon)) \geq F_{k x_{2 j-2}, h x_{2 i-1}}\left(\phi^{2}(\varepsilon)\right) \\
& =F_{f x_{2 i-2}, g x_{2 j-3}}\left(\phi^{2}(\varepsilon)\right) \geq F_{f x_{0}, g x_{2 j-1-2 i}}\left(\phi^{2 i}(\varepsilon)\right) \\
& \geq \sup _{t<\phi^{2 i}(\varepsilon)} \inf _{n, k \in N} F_{y_{n}, y_{k}}(t)=D_{\left\{y_{n}\right\}_{n=1}^{\infty}}\left(\phi^{2 i}(\varepsilon)\right) .
\end{aligned}
$$

Since $\left\{y_{n}\right\}_{n \in N}$ is probabilistically bounded, letting $i \rightarrow \infty$ and $j \rightarrow \infty$, we get 


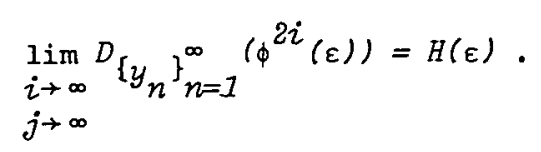

Repeating this procedure we can prove a similar result for $m=2 i-1$ and $p=2 j$.

If $m$ and $p$ are both even or both odd, we proceed as follows.

$$
\begin{aligned}
& F_{y_{2 i}, y_{2 j}}(\varepsilon) \geq t\left(F_{y_{2 i}, y_{2 i+1}}(\varepsilon / 2), F_{y_{2 i+1}, y_{2 j}}(\varepsilon / 2)\right) \rightarrow \\
& \rightarrow t(H(\varepsilon), H(\varepsilon))=H(\varepsilon) \text {, } \\
& F_{y_{2 i-1}, y_{2 j-1}}(\varepsilon) \geq t\left(F_{y_{2 i-1}, y_{2 i}}(\varepsilon / 2), F_{y_{2 i}, y_{2 j-1}}(\varepsilon / 2)\right) \rightarrow \\
& \rightarrow t(H(\varepsilon), H(\varepsilon)) \rightarrow H(\varepsilon)
\end{aligned}
$$

if $i \rightarrow \infty$ and $j \rightarrow \infty$, for all $\varepsilon>0$.

Thus we have proved that $\left\{y_{n}\right\}_{n \in N}$ is a Cauchy sequence in $X$ which means that there exists $y^{*} \in X$ such that $\lim _{n \rightarrow \infty} y_{n}=y^{*}$.

To establish that $f y^{*}=g y^{*}=h y^{*}=k y^{*}$, we proceed as follows.

$$
\begin{aligned}
f y^{*} & =f \lim _{n \rightarrow \infty} k x_{2 n}=\lim _{n \rightarrow \infty} f k x_{2 n}=\lim _{n \rightarrow \infty} k f x_{2 n}= \\
& =k \lim _{n \rightarrow \infty} f x_{2 n}=k y^{*} \\
g y^{*} & =g \lim _{n \rightarrow \infty} h x_{2 n+1}=\lim _{n \rightarrow \infty} g h x_{2 n+1}=\lim _{n \rightarrow \infty} h g x_{2 n+1} \\
& =h \lim _{n \rightarrow \infty} g x_{2 n+1}=h y^{*} .
\end{aligned}
$$

Since

$$
\begin{aligned}
& F_{f y^{*}, g y^{*}}(\varepsilon) \geq F_{k y^{*}, h y^{*}}(\phi(\varepsilon))=F_{f y^{*}, g y^{*}}(\phi(\varepsilon)) \geq \ldots \\
& \ldots \geq F_{f y^{*}, g y^{*}}\left(\phi^{n}(\varepsilon)\right) \rightarrow H(\varepsilon) \text { for all } \varepsilon>0 .
\end{aligned}
$$

we have proved that $f y^{*}=g y^{*}=k y^{*}=h y^{*}$.

The point $f y^{*}$ is a fixed point for the mappings $f, g, h, k$. We shall show this for the mapping $f$; the proof for the mappings $g, h, k$ is analogous. 


$$
\begin{aligned}
& F_{f f y^{*}, f y^{*}}(\varepsilon)=F_{f f y^{*}, g y^{*}}(\varepsilon) \geq F_{k f y^{*}, h y^{*}}(\phi(\varepsilon))= \\
& =F_{f k y^{*}, h y^{*}}(\phi(\varepsilon))=F_{f f y}^{*}, g y^{*}(\phi(\varepsilon)) \geq \ldots \\
& \ldots \geq F_{f f y^{*}, g y^{*}}\left(\phi^{n}(\varepsilon)\right) \rightarrow H(\varepsilon) \text { for } n \rightarrow \infty \text {, }
\end{aligned}
$$

for all $\varepsilon>0$, which means that $f y^{*}$ is a common fixed point for the mappings $f, g, h$, and $k$.

If we suppose that there exists another common fixed point $z \in X$, we get

$$
\begin{aligned}
& F_{f y^{*}, z}(\varepsilon)=F_{f f y^{*}, g z}(\varepsilon) \geq F_{k f y}, h z \\
& =F_{f f y^{*}, g z}(\phi(\varepsilon)) \geq \ldots \geq F_{f y^{*}, z}\left(\phi^{n}(\varepsilon)\right) \rightarrow H(\varepsilon)
\end{aligned}
$$

for $n \rightarrow \infty$ for all $\varepsilon>0$, which means that $f y^{*}$ is a unique common fixed point for the mappings $f, g, h$ and $k$.

This completes the proof of Theorem 1 .

THEOREM 2. If in Theorem 1 the T-norm $t$ satisfies $t(a, b) \geq$ $t_{m}(a, b)=\max \{a+b-1,0\}$, for $a l l a, b \in[0,1]$, then the condition that $f, g, h$ and $k$ are continuous can be replaced by the condition that at least one of the moppings $f, g, h$ and $k$ is continuous.

Proof. The continuity of the mappings $f, g, h$ and $k$ was necessary only to prove that $f y^{*}=g y^{*}=h y^{*}=k y^{*}$.

Now, we assume that at least one of the mappings, say $k$, is continuous. By the commutativity of $f$ and $k$ we have

$$
\lim _{n \rightarrow \infty} k^{2} x_{2 n}=\lim _{n \rightarrow \infty} f k x_{2 n}=k y^{*}
$$

Then

$$
F_{f k x_{2 n}, g x_{2 n+1}}(\varepsilon) \geq F_{k^{2} x_{2 n}, h x_{2 n+1}}(\phi(\varepsilon)), \text { for all } \varepsilon>0 .
$$

Letting $n \rightarrow \infty$, we get (only if $t \geq t_{m}$, see [9])

$$
F_{k y^{*}, y^{*}}(\varepsilon) \geq F_{k y^{*}, y^{*}}(\phi(\varepsilon)) \text {, for all } \varepsilon>0 \text {, }
$$

and so $k y^{*}=y^{*}$. 
Further

$$
F_{f y^{*}, g x_{2 n+1}}(\varepsilon) \geq F_{k y^{*}, h x_{2 n+1}}(\phi(\varepsilon))=F_{y^{*}, h x_{2 n+1}}(\phi(\varepsilon)),
$$

for all $\varepsilon>0$. When $n \rightarrow \infty$ we have: $E_{f y^{*}, y^{*}}(\varepsilon) \geq F_{y^{*}, y^{*}}(\phi(\varepsilon))=H(\varepsilon)$ for all $\varepsilon>0$, that is $f y^{*}=y^{*}$.

From the condition that $f(X) \subset h(X)$, it follows that there exists $z^{*} \epsilon X$ such that $h z^{*}=y^{*}$. Then

$$
\begin{aligned}
F_{y^{*}, g z^{*}}(\varepsilon) & =F_{f y^{*}, g z^{*}}(\varepsilon) \geq F_{k y^{*}, h z^{*}}(\phi(\varepsilon))= \\
& =F_{y^{*}, y^{*}}(\phi(\varepsilon))=H(\varepsilon)
\end{aligned}
$$

for all $\varepsilon>0$, which means that $g z^{*}=y^{*}$. Since $g$ and $h$ commute, the equality $g h z^{*}=g y^{*}=h g z^{*}$ implies that $g y^{*}=h y^{*}$.

Thus

$$
E_{y^{*}, g y^{*}}(\varepsilon)=F_{f y^{*}, g y^{*}}(\varepsilon) \geq F_{k y^{*}, h y^{*}}(\phi(\varepsilon))=F_{y^{*}, g y^{*}}(\phi(\varepsilon)),
$$

for all $\varepsilon>0$, that is $y^{*}=g y^{*}$.

So, we have proved that $y^{*}$ is a fixed point for $f, g, h$ and $k y^{*}=f y^{*}=g y^{*}=k y^{*}=h y^{*}$.

If we suppose that $f, g$ or $h$ is continuous, the proof is similar, so it is omitted.

The other parts of the proof of this Theorem are the same as for Theorem 1 .

\section{Common fixed point in metric spaces.}

If $(M, d)$ is a metric space, then the metric $d$ induces a mapping $F: M \times M \rightarrow \Delta$, where $F(x, y), x, y \in M$, is defined by $F(x, y)(\varepsilon)=$ $F_{x, y}(\varepsilon)=H(\varepsilon-d(x, y)), \varepsilon \in R^{+}$. Further if $t:[0,1] \times[0,1] \rightarrow[0,1]$ is defined by $t(a, b)=\min \{a, b\}$, then $(M, F, t)$ is a Menger space. It is complete if it is complete with respect to the metric $d$. The space $(M, F, \min )$ so obtained will be called the induced Menger space. Using some results stated in the preceding section and the next two lemmas we whall study the existence and uniqueness of common fixed points in metric spaces. 
Throughout this section let $(M, d)$ be a complete metric space, $h: M \rightarrow M, k: M \rightarrow M, f: M \rightarrow h(M)$ and $g: M \rightarrow k(M)$ continuous mappings, such that $f$ commutes with $k$ and $g$ commutes with $h$. Further let the following inequality hold

$$
d(f x, g y) \leq \psi(d(k x, h y))
$$

for all $x, y \in M$, where $\psi$ is a function, $\psi: \mathbb{R}^{+} \rightarrow \mathbb{R}^{+}$.

Let us introduce the following conditions on the mapping $\psi$

$\psi$ is increasing and $\psi^{-1}$ is right upper semicontinuous, $\psi(t)<t$ for all $t \in R^{+}$ $\lim (t-\psi(t))=\infty$. $t \rightarrow \infty$

$\psi$ is increasing, right continuous, for any real number $q \in[0, \infty)$ there exists a real number $t(q) \in[0, \infty)$ such that $t(q)$ is an upper bound of the set $\left\{t \in[0, \infty): t-\psi^{2}(t) \leq q\right\}$, and $\lim _{n \rightarrow \infty} \psi^{n}(t(q))=0$.

Since $f: M \rightarrow h(M)$ and $g: M \rightarrow k(M)$, it is obvious that for every $x_{1} \in M$ we can form the sequence $\left\{y_{n}\right\}_{n \in N}$, by

$$
y_{2 n}=f x_{2 n}=h x_{2 n+1}, y_{2 n+1}=g x_{2 n+1}=k x_{2 n+2} .
$$

Now we prove the following lemma.

LEMMA 1. If $\psi$ satisfies condition $(i)$, then the sequence $\left\{y_{n}\right\}_{n \in N}$ defined above is boronded

Proof. We shall show that

$$
\sup _{\ell \in N} \sup _{1 \leq m, p \leq \ell} d\left(y_{m}, y_{p}\right)<\infty
$$

which means that $\left\{y_{n}\right\}_{n \in N}$ is a bounded set. If $m=2 i$ and $p=2 j-1$ we have

$$
\begin{aligned}
& d\left(y_{2 i}, y_{2 j-1}\right) \leq \psi d\left(y_{2 i-1}, y_{2 j-2}\right) \leq \\
& \leq \psi^{2} d\left(y_{2 i-2}, y_{2 j-3}\right)<\psi d\left(y_{2 i-2}, y_{2 j-3}\right)
\end{aligned}
$$


that is

$$
\begin{gathered}
\sup _{r \leq i, j \leq n} d\left(y_{2 i}, y_{2 j-1}\right) \leq \psi \sup _{n-1 \leq i, j \leq n-1} d\left(y_{2 i}, y_{2 j-1}\right)^{\prime} \\
\leq \psi \sup _{r-1 \leq i, j \leq n} d\left(y_{2 i}, y_{2 j-1}\right) .
\end{gathered}
$$

We know that

$$
\sup _{1 \leq i, j \leq n} d\left(y_{2 i}, y_{2 j-1}\right) \leq d\left(y_{1}, y_{2}\right)+\sup _{2 \leq i, j \leq n} d\left(y_{2 i}, y_{2 j-1}\right) .
$$

Putting $r=2$ in (2) we get

$$
\begin{gathered}
\sup _{2 \leq i, j \leq n} d\left(y_{2 i}, y_{2 j-1}\right) \leq \psi \sup _{1 \leq i, j \leq n-1} d\left(y_{2 i}, y_{2 j-1}\right) \leq \\
\leq \psi \sup _{1 \leq i, j \leq n} d\left(y_{2 i}, y_{2 j-1}\right) .
\end{gathered}
$$

Using the last inequality and (3) we have

$$
\sup _{1 \leq i, j \leq n} d\left(y_{2 i}, y_{2 j-1}\right) \leq d\left(y_{1}, y_{2}\right)+\psi \sup _{1 \leq i, j \leq n} d\left(y_{2 i}, y_{2 j-1}\right) .
$$

If we suppose that

$$
\sup _{1 \leq i, j \leq n} d\left(y_{2 i}, y_{2 j-1}\right)=\infty \text { for } n \rightarrow \infty \text {, }
$$

then from condition (i) and the last inequality we get

$$
\infty=\sup _{1 \leq i, j \leq n} d\left(y_{2 i}, y_{2 j-1}\right)-\psi \sup _{1 \leq i, j \leq n} d\left(y_{2 i}, y_{2 j-1}\right) \leq d\left(y_{1}, y_{2}\right)
$$

which is a contradiction. So, we have proved that

$$
\sup _{n \in N} \sup _{1 \leq i, j \leq n} d\left(y_{2 i}, y_{2 j-1}\right)<\infty \text {. }
$$

Since

$$
\begin{gathered}
\sup _{n \in N} \sup _{1 \leq i, j \leq n} d\left(y_{2 i}, y_{2 j}\right) \leq \sup _{n \in N} \sup _{1 \leq i \leq n} d\left(y_{2 i}, y_{2 i+1}\right)+ \\
\quad \sup _{n \in N} \sup _{1 \leq i, j \leq n} d\left(y_{2 i+1}, y_{2 j}\right)<\infty+\infty=\infty,
\end{gathered}
$$

and

$$
\begin{gathered}
\sup _{n \in N} \sup _{1 \leq i, j \leq n} d\left(y_{2 i-1}, y_{2 j-1}\right) \leq \sup _{n \in N} \sup _{1 \leq i \leq n} d\left(y_{2 i-1}, y_{2 i}\right)+ \\
\quad+\sup _{n \in N} \sup _{1 \leq i, j \leq n} d\left(y_{2 i,}, y_{2 j-1}\right)<\infty+\infty=\infty
\end{gathered}
$$


we have proved that $\left\{y_{n}\right\}_{n \in N}$ is bounded.

LEMMA 2. Let $(M, d)$ be a metric space and $(M, F, m i n l$ be an induced probabilistic metric space. If the inequality (**) holds in $(M, d)$, then the inequality $(*)$ holds in $(M, F, \min )(\psi$ is increasing and $\phi=\psi^{-1}$ ).

Proof. The mapping $\psi$ is increasing and this implies the existence of the increasing mapping $\psi^{-1}=\phi$. To establish that condition $(* *)$ implies condition (*) we prove the next implication:

$$
d(f x, g y) \leq \psi d(h x, k y) \Rightarrow F_{f x, g y}(\varepsilon) \geq F_{h x, k y}\left(\psi^{-1}(\varepsilon)\right) .
$$

Now, we proceed as follows:

$$
\begin{aligned}
& d(f x, g y) \leq \psi d(h x, k y) \\
& \Rightarrow \varepsilon-d(f x, g y) \geq \varepsilon-\psi d(h x, k y) \\
& \Rightarrow H(\varepsilon-d(f x, g y)) \geq H(\varepsilon-\psi d(h x, k y)) \\
& \Rightarrow H(\varepsilon-d(f x, g y)) \geq H\left(\psi^{-1}(\varepsilon)-d(h x, k y)\right) \\
& \Rightarrow F_{f x, g y}(\varepsilon) \geq F_{h x, k y}\left(\psi^{-1}(\varepsilon)\right) .
\end{aligned}
$$

This completes the proof of Lemma 3.

We shall use Theorem 1 and the last two lemmas for the proof of the next Theorem in which we consider the existence and uniqueness of common fixed points for the selfmappings of the metric space $(M, d)$.

THEOREM 3. Let $(M, d)$ be a complete metric space $h: M+M, k: M+M$, $f: M \rightarrow h(M)$ and $g: M \rightarrow k(M)$, where one of these mappings is continuous, $f$ cormutes with $k$ and $g$ commutes with $h$. If inequality $(* *)$ holds, where $\psi: \boldsymbol{R}^{+} \rightarrow \boldsymbol{R}^{+}$is a function for which condition (i) holds, then there exists a unique cormon fixed point for the mappings $f, g, h$ and $k$.

Proof. If $\phi=\psi^{-1}$ then the mapping $\phi$ satisfies the condition of Theorem 1, [2]. Lerma 1 and Lemma 2 provide the other conditions of Theorem 1, so this theorem is proved.

THEOREM 4. If the mopping $\psi:[0, \infty) \rightarrow[0, \infty)$ satisfies condition (ii), then the conclusion of the last Theorem stizl holds. 
Proof. The proof has four steps. We shall prove that

1. $\left\{y_{n}\right\}$ is a Cauchy sequence and $\lim _{n \rightarrow \infty} y_{n}=y^{*}$,

2. $f y^{*}=g y^{*}=h y^{*}=k y^{*}=u$,

3. $u$ is a common fixed point for $f, g, h$ and $k$,

4. $u$ is a unique common fixed point.

1. We shall show that

$$
\lim _{\substack{m \rightarrow \infty \\ p \rightarrow \infty}} d\left(y_{m}, y_{p}\right)=0 .
$$

If $m=2 i$ and $p=2 j-1$ we have

(4)

$$
d\left(y_{2 i}, y_{2 j-1}\right) \leq \psi d\left(y_{2 i-1}, y_{2 j-2}\right) \leq \psi^{2} d\left(y_{2 i-2}, y_{2 j-3}\right)
$$

That is

$$
\begin{gathered}
\sup _{r \leq i, j \leq n} d\left(y_{2 i}, y_{2 j-1}\right) \leq \psi^{2} \sup _{r-1 \leq i, j \leq n-1} d\left(y_{2 i}, y_{2 j-1}\right) \\
\leq \psi^{2} \sup _{r-1 \leq i, j \leq n} d\left(y_{2 i}, y_{2 j-1}\right) .
\end{gathered}
$$

From (5) and (3) (letting $r=2$ ) we get

$$
\begin{gathered}
\sup _{1 \leq i, j \leq n} d\left(y_{2 i}, y_{2 j-1}\right) \leq d\left(y_{1}, y_{2}\right) \\
+\psi^{2} \sup _{1 \leq i, j \leq n} d\left(y_{2 i}, y_{2 j-1}\right) .
\end{gathered}
$$

Letting the real number $q=d\left(y_{1}, y_{2}\right)$ and using condition (ii) it follows, from the last inequality, that there exists a real number $t(q) \in[0, \infty)$ such that

$$
\sup _{1 \leq i, j \leq n} d\left(y_{2 i}, y_{2 j-1}\right) \leq t(q) \text {. }
$$

Taking $r=2$ in (4) again, and considering the inequality we have

$$
\sup _{2 \leq i, j \leq n} d\left(y_{2 i}, y_{2 j-1}\right) \leq \psi^{2}(t(q)) \text {. }
$$

Repeating this procedure, for any positive integers $r, n$ we obtain

$$
\sup _{r \leq i, j \leq n} d\left(y_{2 i}, y_{2 j-1}\right) \leq \psi^{2 r-2}(t(q)) \text {. }
$$


Letting $r \rightarrow \infty$ (hence $n \rightarrow \infty$ ) in (6), from condition (ii) we get

$$
0 \leq \lim _{r \rightarrow \infty} \sup _{r \leq i, j \leq n} d\left(y_{2 i}, y_{2 j-1}\right) \leq \lim _{r \rightarrow \infty} \psi^{2 r-2}(t(q))=0
$$

If $m$ and $p$ are both even or both are odd, we proceed as follows

$$
\begin{aligned}
& \lim _{r \rightarrow \infty} \sup _{r \leq i, j \leq n} d\left(y_{2 i}, y_{2 j}\right) \leq \lim _{r \rightarrow \infty} \sup _{r \leq i, j \leq n} d\left(y_{2 i}, y_{2 i+1}\right) \\
& +\lim _{r \rightarrow \infty} \sup _{r \leq i, j \leq n} d\left(y_{2 i+1}, y_{2 j}\right)=0+0=0,
\end{aligned}
$$

and

$$
\begin{aligned}
\lim _{r \rightarrow \infty} & \sup _{r \leq i, j \leq n} d\left(y_{2 i-1}, y_{2 j-1}\right) \leq \lim _{r \rightarrow \infty} \sup _{r \leq i, j \leq n} d\left(y_{2 i-1}, y_{2 i}\right) \\
& +\lim _{r \rightarrow \infty} \sup _{r \leq i, j \leq n} d\left(y_{2 i}, y_{2 j-1}\right)=0+0=0 .
\end{aligned}
$$

This shows that $\left\{y_{n}\right\}_{n \in N}$ is a Cauchy sequence in the complete metric space $(M, d)$ and so there exists $y^{*} \in X$ such that $\lim _{n \rightarrow \infty} y_{n}=y^{*}$.

2. From the continuity of the mapping $f, g, h$ and $k$, in the same way as in Theorem 1 , we get that $f y^{*}=k y^{*}$ and $g y^{*}=h y^{*}$. To prove that $f y^{*}=g y^{*}$ we proceed as follows:

$$
\begin{aligned}
& d\left(f y^{*}, g y^{*}\right) \leq \psi d\left(h y^{*}, k y^{*}\right)=\psi d\left(f y^{*}, g y^{*}\right) \\
& <\psi^{2} d\left(h y^{*}, k y^{*}\right)=\psi^{2} d\left(f y^{*}, k y^{*}\right) .
\end{aligned}
$$

From (ii) if $q=0$ there exists $t(q) \in[0, \infty)$ such that

$$
d\left(f y^{*}, g y^{*}\right) \leq t(0) \Rightarrow \psi^{n} d\left(f y^{*}, g y^{*}\right) \leq \psi^{n}(t(0)) \text {. }
$$

Since

$$
d\left(f y^{*}, g y^{*}\right) \leq \psi^{n} d\left(f y^{*}, g y^{*}\right) \leq \psi^{n}(t(0)) \rightarrow 0 \text { for } n \rightarrow \infty
$$

we have that $f y^{*}=g y^{*}$, that is $f y^{*}=g y^{*}=h y^{*}=k y^{*}=u$.

3. We shall prove that $f u=u$ (similarly, we can prove the same for the mappings $g, h$ and $k$ ).

$$
\begin{gathered}
d(f u, u)=d\left(f u, g y^{*}\right) \leq \psi d\left(h u, k y^{*}\right)=\psi d\left(f u, g y^{*}\right) \\
\quad \leq \psi^{2} d(f u, u) \leq \ldots \leq \psi^{n} d(f u, u) .
\end{gathered}
$$

putting $q=0$ from (ii) there exists $t(0) \in[0, \infty)$ such that 


$$
\begin{aligned}
& d(f u, u) \leq t(0) \Rightarrow \psi^{n} d(f u, u) \leq \psi^{n}(t(0)) \\
& \Rightarrow d(f u, u) \leq \psi^{n} d(f u, u) \leq \psi^{n}(t(0)) \stackrel{n \rightarrow 0}{\rightarrow} 0 \Rightarrow f u=u .
\end{aligned}
$$

4. If we suppose that the point $v \in X$ is also a common fixed point for the mappings $f, g, h$ and $k$ we get

$$
\begin{aligned}
& d(u, v)=d(f u, g v) \leq \psi(h u, k v)=\psi(f u, g v) \leq \ldots \\
& \ldots \leq \psi^{n} d(u, v)
\end{aligned}
$$

so that if $q=0$, then there exists $t(0)$ such that $d(u, v) \leq t(0) \Longrightarrow$ $d(u, v) \leq \psi^{n} d(u, v) \leq \psi^{n}(t(0)) \stackrel{n \rightarrow \infty}{\rightarrow} 0 \Rightarrow u=v$.

6. Application and examples.

First, we shall give an application of Theorem 1 in a well known Menger space - E-space.

An ordered pair $(E, F)$ is called an $E$-space over $(M, d)$ if $E$ is the collection of all random variables from a complete probability measure space $(\Omega, A, P)$ into a separable complete metric space $(M, d)$ such that for every $\xi(\omega), n(\omega) \in E$ and every $t \in R$

$$
\{\omega \in \Omega: \quad d(\xi(\omega), n(\omega))<t\} \in A
$$

and $F$ is a mapping from $E \times E$ into $\Delta$ (the set of all distribution functions) defined by $F(\xi, \eta)=F_{\xi, n}$, where

$$
F_{\xi, \eta}(t)=P\{\omega \in \Omega: d(\xi(\omega), \eta(\omega))<t\}
$$

for every $t \in R$. As usual, the random variables in $E$ which differ at most on a set of $P$-measure zero are identified.

As sherwood [10] pointed out, $(E, F)$ is an $E$-space over $(M, d)$ and $\left(E, F, t_{m}\right)$ is a Menger space, where the $T$-norm $t_{m}(a, b)=\max \{a+b-1,0\}$, $a, b \in[0,1]$. If $(M, d)$ is a complete metric space, then $\left(E, F, t_{m}^{\prime}\right.$ is complete.

A mapping $f: \Omega \times M \rightarrow M$ is a random operator, if for any $x \in M, y(\omega)=f(\omega, x)$ is a random variable. A random operator $f$ is continuous if for each $\omega \in \Omega, f(\omega, \cdot)$ is continuous in the topology induced by the metric $d$. 
A random variable $\xi(\omega) \in E$ is a random fixed point of the random operator $f(\omega, \cdot)$ if $\xi(\omega)=f(\omega, \xi(\omega))$ for all $\omega \in \Omega$.

If $f$ is a continuous random operator, then $\xi(\omega) \in E$ implied $f(\omega, \xi(\omega)) \in E$.

It is obvious that all mappings which satisfy the conditions of Theorem 2, have a unique common fixed point.

Let us consider the next simple example.

EXAMPLE 1. Let $\left(E, F, t_{m}\right)$ be a Menger space, where $E$ is the collection of random variables from $(\Omega, A, P)$ into $R$.

Let

$$
f(\xi(\omega))=g(\xi(\omega))=\frac{1}{2}(\xi(\omega)+1)
$$

and

$$
h(\xi(\omega))=k(\xi(\omega))=2 \xi(\omega)-1 .
$$

since $f, g, h$ and $k$ are linear mappings, they are continuous and surjective. Further we have that $f^{-1}=h$ and $g^{-1}=k$, hence $f$ commutes with $h$ and $g$ commutes with $k$. If we chose a mapping $\phi: R^{+} \rightarrow R^{+}$such that $t<\phi(t)<4 t(\phi(t)=2 t$, for instance) then the next inequalities hold for all $\xi(\omega), n(\omega) \in E$ and all $\varepsilon \in R^{+}$.

$$
\begin{aligned}
& P\{\omega \in \Omega:|\xi(\omega)-n(\omega)|<2 \varepsilon\} \\
& \geq P\left\{\omega \in \Omega:|\xi(\omega)-n(\omega)|<\frac{\phi(\varepsilon)}{2}\right\}, \\
& P\left\{\omega \in \Omega:\left|\frac{1}{2}(\xi(\omega)+1)-\frac{1}{2}(n(\omega)+1)\right|<\varepsilon\right\} \\
& \geq P\{\omega \in \Omega:|2 \xi(\omega)-1-(2 n(\omega)-1)|<\phi(\varepsilon)\}, \\
& P\{\omega \in \Omega: \mid f(\xi(\omega))-g(n(\omega))<\varepsilon\} \\
& \geq P\{\omega \in \Omega:|k(\xi(\omega))-h(n(\omega))|<\phi(\varepsilon), \\
& F_{f(\xi(\omega)), g(\eta(\omega))}(\varepsilon) \geq F_{k(\xi(\omega)), h(\eta(\omega))}(\phi(\varepsilon)) .
\end{aligned}
$$

Now, we can form the sequence $\left\{y_{n}(\omega)\right\}$ as in Theorem 1 . Let $x_{1}(\omega) \in E$ be a random variable such that for some $A \in R^{+}$ $P\left\{\omega \in \Omega:\left|x_{1}(\omega)\right|<A\right\}=1$. One can define the sequence $\left\{y_{n}(\omega)\right\}$ in the 
following way (as in Theorem 1 ).

$$
y_{n}(\omega)=\frac{1}{2^{2 n-1}} x_{1}(\omega)+\sum_{i=1}^{2 n-1} \frac{1}{2^{i}} .
$$

To prove that $\left\{y_{n}(\omega)\right\}_{n \in N}$ is probabilistically bounded we shall show that

$$
\sup _{\varepsilon>0} \inf _{n, k \in N} F_{n}(\omega), y_{k}(\omega)(\varepsilon)=1
$$

Without loss of generality, we can suppose that in the next relation $n<k$.

$$
\begin{aligned}
& \sup _{\varepsilon>0} \inf _{n, k \in N} F_{y_{n}(\omega), y_{k}(\omega)}(\varepsilon)= \\
= & \sup _{\varepsilon>0} \inf _{n, k \in N} P\left\{\omega \in \Omega:\left|y_{n}(\omega)-y_{k}(\omega)\right|<\varepsilon\right\} \\
= & \sup _{\varepsilon>0} \inf _{n, k \in N} P\left\{\omega \in \Omega: \mid\left(\frac{1}{2^{2 n-1}}-\frac{1}{2^{2 k-1}}\right) x_{1}(\omega)\right. \\
+ & \left.\sum_{i=2 n-1}^{2 k-1} \frac{1}{2^{i}} \mid<\varepsilon\right\} \\
\geq & \sup _{\varepsilon>0} \inf _{, k \in N} P\left\{\omega \in \Omega:\left|x_{1}(\omega)+1\right|<\varepsilon\right\} \\
\geq & P\left\{\omega \in \Omega:\left|x_{1}(\omega)+1\right|<A+1\right\}=1 .
\end{aligned}
$$

Since all the conditions of Theorem 2 are satisfied, $f$ has a unique fixed point $y^{*}(\omega)=\lim _{n \rightarrow \infty} y_{n}(\omega)$, and it is easy to find it. If $n \rightarrow \infty$ the distribution function of random variable $y^{*}(\omega)$ is

$$
\begin{aligned}
F_{y^{*}}(\varepsilon) & =\lim _{n \rightarrow \infty} P\left\{\omega \in \Omega:\left(\frac{1}{2^{2 n-1}} x_{1}(\omega)+\sum_{i=1}^{2 n-1} \frac{1}{2^{i}}\right)<\varepsilon\right\} \\
& = \begin{cases}0 & \varepsilon \leq 1 \\
1 & \varepsilon>1 .\end{cases}
\end{aligned}
$$

In Examples 2, 3 and 4 we shall show that we can not omit any of the conditions of Theorem 3 (or Theorem 2).

EXAMPLE 2. Let $M=[0, \infty), f(x)=g(x)=h(x)=0, k(x)=|1-x|$, $\psi(t)=\ln (t+1)$. All the conditions of Theorem 3 are satisfied except for the commutativity of $f$ and $k$, and $f, g, h$ and $k$ have no 
common fixed point.

EXAMPLE 3. If $M=[0,1], f(x)=\left\{\begin{array}{l}1 / 4, x=0 \\ x / 4, x \neq 0\end{array}, g(x)=\frac{x}{4}\right.$,

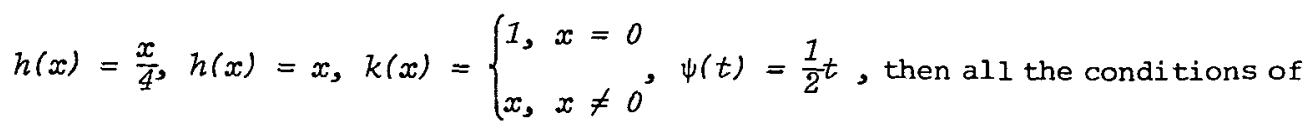
Theorem 3 are satisfied except $g(M) \subset k(M)\left(g(M)=\left[0, \frac{1}{4}\right], k(M)=(0,1]\right)$, and $f, g, h$ and $k$ have no common fixed point.

EXAMPLE 4. If $M=[0,1], f(x)=g(x)=\left\{\begin{array}{l}1 / 2, x=0 \\ x / 4, x \neq 0\end{array} k(x)=\right.$ $h(x)=\left\{\begin{array}{c}1, x=0 \\ x / 2, x \neq 0\end{array}, \psi(t)=\frac{1}{2} t\right.$, then all the conditions of Theorem 3 are satisfied except that $f, g, h$ and $k$ are all discontinuous, and $f, g, h$ and $k$ have no fixed point.

\section{References}

[1] Shih-Sen Chang, "A common fixed point theorem for commuting mappings", Proc. Amer. Math. Soc., 83 (1981), 645-652.

[2] Xie Ping Ding, "New results on common fixed points", Math. Sem. Notes Kobe Univ., 10 (1982), 623-631.

[3] B. Fisher, K. Iseki, "Fixed points for set-valued mappings on complete and compact metric space", Mat. Japon. 28 (1983), 639-646.

[4] B. Fisher, "Common fixed points of four mappings", Bull. Inst. Math. Acad. Sinica, 11 (1983), 103-113.

[5] 0 . Hadzic, "Common fixed point theorems for family of mappings in complete metric spaces", Math. Jopon. 29 (1984), 124-134.

[6] o. Hadzic, Fixed point theory in topological vector spaces, (Univ. of Novi Sad, Inst. of Math., Novi Sad, 1984).

[7] S. Kasahara, B.E. Rhoades, "Common fixed point theorems in compact metric spaces", Math. Jopon. 23, No. 2, (1978), 227-229.

[8] B.E. Rhoades, "A comparison of various definitions of contractive mappings", Trons. Amer. Math. Soc., 226 (1977), 257-290.

[9] B. Schweizer, A. Sklar, "Statistical metric spaces", Pacific $J$. Math., 10 (1960), 313-324. 
[10] H. Sherwood, "On E-spaces and their relation to other classes of probabilistic metric spaces", J. London Math. Soc., 44 (1969), 441-449.

[11] M. Stojakovic, "Fixed point theorems in probabilistic metric spaces", Kobe J. Math., 2 (1985), 1-9.

[12] M. Stojaković, "A common fixed point for the commuting mappings", Indican J. Pure App Z. Math., 17 (1986), 446-475.

University of Novi Sad

Faculty of Technical Sciences

Department of Mathematics

Veljka vlahovića 3

21000 Novi Sad

Yugoslavia 